\title{
Bone as an Endocrine Organ
}

\author{
Anyonya R Guntur, $\mathrm{PhD}^{1}$ and Clifford J Rosen, $\mathbf{M D}^{1}$ \\ ${ }^{1}$ Center for Clinical and Translational Research, Maine Medical Center Research Institute, 81 \\ Research Drive Scarborough, Maine 04074
}

\begin{abstract}
Bone has classically been viewed as an inert structure that is necessary for mobility, calcium homeostasis, and maintenance of the hematopoietic niche. Recent advances in bone biology using complex genetic manipulations in mice have highlighted the importance of bone not only as a structural scaffold to support the human body, but also as a regulator of a number of metabolic processes that are independent of mineral metabolism. These advances point to the skeleton as an endocrine organ that modulates glucose tolerance and testosterone production by secretion of a bone-specific protein, osteocalcin. This review will detail how bone has emerged as a bona-fide endocrine "gland", and with that, the potential therapeutic implications that could be realized for this hormone-secreting tissue.
\end{abstract}

\section{Introduction}

The role of an endocrine organ is to regulate distant function through the secretion of a peptide or steroid hormone. Classically, bone has been considered as a structural organ that develops through the actions of specialized cells, particularly chondrocytes and osteoblasts. Osteoblasts synthesize bone matrix and ultimately become entombed within that matrix to become osteocytes, cells that act as sensors for gravitational forces as well as mediators for intercellular communications within the remodeling unit (1). Bone is dynamic and is constantly undergoing remodeling, which is enabled by another group of specialized cells, the osteoclasts, as well as osteoblasts and osteocytes. Osteoclasts secrete protons and enzymes responsible for the ultimate resorption of bone. A disruption or imbalance in these processes can lead to either an increase or decrease in bone mineral density that may be detrimental to skeletal strength (2).

Recent advances in the use of genetic engineering have shed light on novel pathways and mechanisms through which bone might function as an endocrine organ. Previously, it had been reported that osteocytes secreted a bone-specific protein, sclerostin that could act to inhibit osteoblast differentiation in a paracrine fashion (3). In addition, it was known that osteocytes synthesized and secreted FGF-23 into the circulation, an important factor that regulates 1-alpha-hydroxylase, serum phosphate, and PTH (4). Those studies supported an endocrine role for the skeleton in regulating mineral metabolism, but few believed that bone could act to modulate metabolic homeostasis. However, within the last half decade, tremendous strides in utilization of mouse models have helped investigators focus on osteocalcin, a bone-specific, non-collagen protein whose function had long been debated but never resolved. The first part of this review will address some of the studies that have been performed to unravel the molecular pathways that are influenced by secretion of osteocalcin,

\footnotetext{
*Address correspondence to: Clifford J Rosen, MD, Center for Clinical and Translational Research, Maine Medical Center Research Institute, 81 Research Drive, Scarborough, Maine 04074, crofen@gmail.com, Phone 207-885-8100, Fax 207-885-8174.

Disclosure

The authors have no multiplicity of Interest to disclose.
} 
with a particular focus on the regulation of glucose homeostasis. The second part of this review will discuss the upregulation of testosterone secretion that is observed with enhanced osteocalcin production and its effect on male fertility in mice.

\section{Osteocalcin and its role in regulating glucose metabolism}

In mammals, utilization of glucose as the major source of energy is necessary for survival. Dysfunction in the regulation and utilization of this source of energy leads to a wide array of metabolic syndromes (5). The increase of blood glucose with loss of insulin sensitivity is a hallmark of type II diabetes and has been of considerable research interest as a majority of the obese population is insulin insensitive. Recent investigations, particularly from the Karsenty group, have identified a crucial role for osteocalcin in regulating insulin metabolism in a hormonal manner. Osteocalcin is made by differentiated osteoblasts and is gamma-carboxylated to enhance attachment to hydroxyapatite $(6,7)$. Its role in skeletal remodeling has been debated for several years, particularly after it was found that deletion of the osteocalcin gene had little effect on the skeletal phenotype, other than a slight increase in bone density (8). As such, this model was ignored for several years until it became clear that osteocalcin is also stored within the skeletal matrix, thereby providing the circulation with a relatively plentiful source of peptide during resorption. As it turns out, both undercarboxylated and carboxylated osteocalcin are released during active resorption from the skeletal matrix. Moreover, a closer examination of the osteocalcin-null mice revealed increased adiposity and a dysfunction in glucose homeostasis/regulation. It was subsequently noted that the adiposity phenotype resulted from changes in undercarboxylated osteocalcin-mediated increases in $\beta$-cell proliferation and insulin sensitivity and secretion. The Karsenty group, in a series of elegant experiments, went on to demonstrate that the relatively strong effects of loss of osteocalcin on glucose homeostasis were due in part to changes in a phosphatase enzyme that binds to the insulin receptor (9). Indeed, osteoblasts express a negative regulator of osteocalcin which is the product of Esp (Ptprv), a tyrosine phosphatase which, through its interaction with the insulin receptor, produces the inactive form of osteocalcin (i.e. carboxylated osteocalcin; osteocalcin is active in its undercarboxylated form). Both germline and conditional deletion of the Esp gene (Esp-/-) demonstrate that the metabolic phenotype of these mice is exactly opposite to that of the osteocalcin knockout. The human ortholog of Esp (OST-PTP, also called osteo-testicular protein tyrosine phosphatase) is not active in humans but recent studies have shown that there are additional tyrosine phosphatases, such as TC-PTP1, that are expressed in osteoblasts. These can regulate osteocalcin activity and glucose homeostasis by acting on the insulin signaling pathway in the osteoblast $(9,10,11)$.

To study this phenomenon, a number of groups (e.g. Karsenty, Clemens, and Kousteni) explored the molecular mechanisms that could be operative. Deletion of FoxO1, which is one of the major FoxOs expressed in bone, has been studied using a conditional knockout mouse to examine the role of this protein exclusively in bone. FoxO1 osteoblast conditional knockout mice have lower blood glucose levels and have higher insulin levels than WT controls. The regulation of Esp gene transcription thus seems to occur by binding of FoxO1 and another osteoblast-specific transcription factor, ATF4, to the promoter region of Esp, leading to its upregulation (12). Subsequently, it was observed that the knockout of ATF4 specifically in osteoblasts leads to a reduction in blood glucose levels, suggesting that there is an increase in the active form of osteocalcin because of the lack of an increase in Esp (13).

Though the Esp knockout displayed the metabolic opposite of the osteocalcin knockout phenotype, the osteoblast-specific knockout of the insulin receptor (IR) has also provided insights into the mechanism through which glucose sensitivity is altered. Utilization of insulin receptor conditional knockout mice (i.e. deletion of the IR only in osteoblasts), in 
which the Cre recombinase necessary to delete the IR is under the control of the collagen1 promoter, has shown that conditional IR-/- mice have increased blood glucose levels and decreased levels of insulin in response to glucose compared to control mice $(14,15)$. Since it was also observed that osteoblast-specific FoxO1 conditional knockout mice show reduced blood glucose levels (the metabolic phenotype is opposite to that of the osteocalcin knockout mice) the deletion of one allele of FoxO1 in the IR knockout mice mostly corrected the phenotype, suggesting that IR in osteoblasts acts through the FoxO1 pathway in regulating glucose homeostasis. Some of the additional genes that IR acts on are the Twist family of transcriptional factors that have been known to act by binding to Runx 2 and thereby negatively regulating osteoblastogenesis. Indeed, the loss of IR in osteoblasts led to an increase in Twist2, which can then down-regulate Runx2 transcriptional activity leading to a decrease in osteocalcin expression and reduced bone formation. Importantly, the Twist family of helix-loop-helix transcriptional factors has been shown to be induced by Wnt signaling during bone development; family members can also act as major negative regulators of bone formation through their actions on master transcriptional regulators of chondrogenesis (16) and osteogenesis (e.g. Sox9, and Runx2).

Thus, through the use of osteoblast-specific knockout mice, there is a strong body of evidence suggesting that glucose homeostasis is controlled by osteocalcin production. The preponderance of evidence that this pathway is necessary in humans and that there is a relationship between the levels of circulating osteocalcin and type II diabetes has been growing exponentially with observational data, although clinical trials of osteocalcin have not been initiated.

\section{Osteocalcin and energy expenditure}

The osteocalcin knockout mice show a decrease in energy expenditure and the Esp bonespecific knockout mice show the exact opposite phenotype (i.e. an increase in energy expenditure and resistance to high-fat diet-induced obesity). The study of individual cell types and the utilization of different substrates will ultimately help identify the extent and contribution of several different cell types (osteoblasts/osteoclasts/osteocytes) in regulating energy expenditure. Another phenotype often seen in the marrow during metabolic stress and changes in substrate utilization is marrow adiposity. The use of anti-diabetic drugs such as rosiglitazone have shown that in mice and in humans there is an increase in marrow adipogenesis and a concurrent loss in bone mineral density (17). Rosiglitazone enhances PPAR $\gamma$ activity which can, in turn, suppress bone formation and increase bone resorption while simultaneously enhancing insulin sensitivity (18). Recent studies have shown that one mechanism could be through the regulation of PPAR $\gamma$ by FGF21, an endocrine factor that is synthesized in liver and white adipose tissue in a diurnal fashion and in response to food restriction $(19,20)$. Studies from our group have also shown that Nocturnin, a deadenylase that can regulate both adipogenesis and osteogenesis, modulates the activity of PPAR $\gamma$ and thereby mediates bone loss in response to rosiglitazone (21). Taken together, these data suggest a close relationship between bone acting as an endocrine organ and as well as a target of adipocyte-generated cytokines. The fact that the bone marrow has increased marrow adipocytes in some conditions such as diabetes, aging, and anorexia nervosa, emphasizes the importance of the interactions between skeletal and metabolic tissues.

\section{Osteocalcin and its role in regulating testosterone}

Osteocalcin may also have another hormonal role, this time as a mediator of testosterone secretion. Osteocalcin was shown to induce testosterone production in leydig cells of the testes both in ex vivo and in vivo studies. Its mode of action was found to be through a novel G protein-coupled receptor (GPCR6A) expressed on the surface of leydig cells. Additional 
evidence implicating GPCR6A, a 7- transmembrane receptor, as a receptor for osteocalcin, was the finding that knockout of this gene in mice phenocopies the osteocalcin knockout metabolic syndrome (22). There have been a number of previous studies that looked at the role of estrogen on bone, but the role of osteocalcin regulating testosterone production was novel and somewhat provocative. For example, the addition of osteocalcin to leydig primary cell cultures provoked an increase in CREB through the GPCR6A receptor. This can then act as a transcriptional factor to regulate the synthesis of the key enzymes involved in testosterone production, such as CYP11a1, Cyp17, and STAR (23). In vivo proof for the cellular studies utilized a number of tissue-specific knockouts, as noted in table 1. Interestingly, a study has shown that Runx2, which controls osteocalcin expression, can also control the expression of CYP11a1 and its shorter isoform that is expressed in osteoblasts. That study showed that upregulation is through direct binding of Runx 2 to the promoter region of CYP11a1 (24). The expression of Runx family of transcriptional factors along with CYP11a1 in the testis (25) is likely more than coincidence and raises some provocative questions about osteocalcin regulation of testosterone, particularly during stages of active bone formation such as pubertal growth.

\section{Conclusion}

For more than 5 decades it had been known that the skeleton was a target of endocrine hormones such as parathyroid hormone and estrogen. Only in the last five years has it become evident that the skeleton reciprocates for being an endocrine target by secreting its own factors to modulate metabolic function. The seminal studies from the Karsenty laboratory began an evolution in our thinking about the role of the skeleton in whole body homeostasis. The use of genetically engineered mice has made possible a new paradigm with potentially important therapeutic implications. It is also likely that other factors may be operative as skeletal endocrine factors, particularly in respect to insulin sensitivity. Development of new drugs that target both the skeleton and adipose tissue are certain to be considered within the realm of this new paradigm. As endocrinologists, this is an exciting time not only for new drugs, but also for going back to the roots of our specialty, i.e. the role of circulating factors in modulating biochemical and genetic pathways.

\section{Acknowledgments}

The work in Dr. Rosen's laboratory is funded by NIH grant AR045433. The authors thank Casey R. Doucette for critical reading of the manuscript.

\section{References}

1. Karsenty G, Wagner EF. Reaching a Genetic and Molecular Understanding of Skeletal Development. Developmental Cell. 2002; 2(4):389-406. [PubMed: 11970890]

2. Teitelbaum SL. Osteoclasts: What Do They Do and How Do They Do It? The American Journal of Pathology. 2007; 170(2):427-435. [PubMed: 17255310]

3. Winkler DG, et al. Osteocyte control of bone formation via sclerostin, a novel BMP antagonist. EMBO J. 2003; 22(23):6267-6276. [PubMed: 14633986]

4. Shimada T, et al. Targeted ablation of Fgf23 demonstrates an essential physiological role of FGF23 in phosphate and vitamin D metabolism. The Journal of Clinical Investigation. 2004; 113(4):561568. [PubMed: 14966565]

5. Grundy SM. Hypertriglyceridemia, insulin resistance, and the metabolic syndrome. The American Journal of Cardiology. 1999; 83(9, Supplement 2):25-29.

6. Hauschka PV, Lian JB, Cole DE, Gundberg CM. Osteocalcin and matrix Gla protein: vitamin Kdependent proteins in bone. Physiological Reviews. 1989; 69(3):990-1047. [PubMed: 2664828] 
7. Hauschka PV, Lian JB, Gallop PM. Direct identification of the calcium-binding amino acid, gamma-carboxyglutamate, in mineralized tissue. Proceedings of the National Academy of Sciences. 1975; 72(10):3925-3929.

8. Ducy P, et al. Increased bone formation in osteocalcin-deficient mice. Nature. 1996; 382(6590): 448-452. [PubMed: 8684484]

9. Lee NK, et al. Endocrine Regulation of Energy Metabolism by the Skeleton. Cell. 2007; 130(3): 456-469. [PubMed: 17693256]

10. Cousin W, Courseaux A, Ladoux A, Dani C, Peraldi P. Cloning of hOST-PTP: the only example of a protein-tyrosine-phosphatase the function of which has been lost between rodent and human. 2004

11. Zee T, Settembre C, Levine RL, Karsenty G. T-Cell Protein Tyrosine Phosphatase Regulates Bone Resorption and Whole-Body Insulin Sensitivity through Its Expression in Osteoblasts. Molecular and Cellular Biology. 2012; 32(6):1080-1088. [PubMed: 22252315]

12. Rached M-T, et al. FoxO1 expression in osteoblasts regulates glucose homeostasis through regulation of osteocalcin in mice. The Journal of Clinical Investigation. 2010; 120(1):357-368. [PubMed: 20038793]

13. Kode A, et al. FoxO1 Protein Cooperates with ATF4 Protein in Osteoblasts to Control Glucose Homeostasis. Journal of Biological Chemistry. 2012; 287(12):8757-8768. [PubMed: 22298775]

14. Ferron M, et al. Insulin Signaling in Osteoblasts Integrates Bone Remodeling and Energy Metabolism. Cell. 2010; 142(2):296-308. [PubMed: 20655470]

15. Fulzele K, et al. Insulin Receptor Signaling in Osteoblasts Regulates Postnatal Bone Acquisition and Body Composition. Cell. 2010; 142(2):309-319. [PubMed: 20655471]

16. Gu S, Boyer TG, Naski MC. Basic helix-loop-helix transcription factor twist1 inhibits the transactivator function of the master chondrogenic regulator Sox9. Journal of Biological Chemistry. 2012

17. Lecka-Czernik B. Bone Loss in Diabetes: Use of Antidiabetic Thiazolidinediones and Secondary Osteoporosis. Current Osteoporosis Reports. 2010; 8(4):178-184. [PubMed: 20809203]

18. Rzonca SO, Suva LJ, Gaddy D, Montague DC, Lecka-Czernik B. Bone Is a Target for the Antidiabetic Compound Rosiglitazone. Endocrinology. 2004; 145(1):401-406. [PubMed: 14500573]

19. Oishi K, Konishi M, Murata Y, Itoh N. Time-imposed daily restricted feeding induces rhythmic expression of Fgf21 in white adipose tissue of mice. Biochemical and Biophysical Research Communications. 2011; 412(2):396-400. [PubMed: 21835167]

20. Wei W, et al. Fibroblast growth factor 21 promotes bone loss by potentiating the effects of peroxisome proliferator-activated receptor $\gamma$. Proceedings of the National Academy of Sciences. 2012; 109(8):3143-3148.

21. Guntur AR, et al. An essential role for the circadian-regulated gene Nocturnin in osteogenesis: the importance of local timekeeping in skeletal homeostasis. Annals of the New York Academy of Sciences. 2011; 1237(1):58-63. [PubMed: 22082366]

22. Pi M, et al. GPRC6A Null Mice Exhibit Osteopenia, Feminization and Metabolic Syndrome. PLoS ONE. 2008; 3(12):e3858. [PubMed: 19050760]

23. Oury F, et al. Endocrine Regulation of Male Fertility by the Skeleton. Cell. 2011; 144(5):796-809. [PubMed: 21333348]

24. Teplyuk NM, et al. The Osteogenic Transcription Factor Runx2 Controls Genes Involved in Sterol/ Steroid Metabolism, Including Cyp11a1 in Osteoblasts. Molecular Endocrinology. 2009; 23(6): 849-861. [PubMed: 19342447]

25. Jeong J-H, et al. Expression of Runx2 transcription factor in non-skeletal tissues, sperm and brain. Journal of Cellular Physiology. 2008; 217(2):511-517. [PubMed: 18636555] 


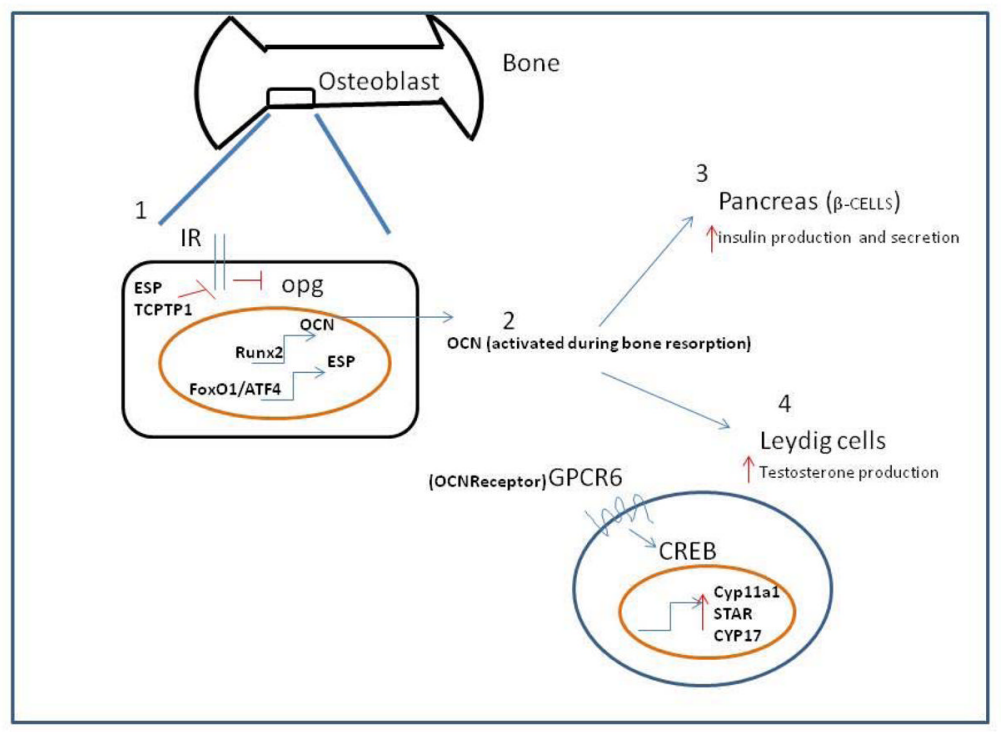

Figure 1.

shows the effects of osteoblast-secreted osteocalcin on glucose homeostasis and its novel role in regulating testosterone production in the leydig cells. In stage $\mathbf{1}$, activation of insulin receptor can negatively regulate the expression of ESP (FoxO1 and AFT4 can regulate its transcription). Insulin signaling can also inhibit OPG (osteoprotegerin), a negative regulator of osteoclastogenesis. Osteocalcin, once activated (2), can act on the pancreas (3) (GPCR6A?) and the testes through the GPCR6A receptor, which activates the CREB signaling pathway leading to an increase in testosterone production through transcriptional upregulation of the various enzymes involved in its biosynthetic pathway (4). OCN osteocalcin, ESP osteo-testicular protein tyrosine phosphatase, OPG (osteoprotegerin), TCPTP1 (T-Cell Protein Tyrosine Phosphatase), GPCR6A (member of family C, G protein coupled receptor), StAR (steroidogenic acute regulatory protein), CYP11a1(cholesterol side chain cleavage enzyme), CYP17(cytochrome P450 17a) 
Table 1

shows the different mouse models that have been described in the paper and the metabolic phenotypes that were observed.

\begin{tabular}{|l|l|l|}
\hline Mouse knockouts & References & Metabolic phenotypes observed \\
\hline Osteocalcin knockout & Ducy et al (8) & Increased blood glucose, decreased insulin levels and obese. \\
\hline $\begin{array}{l}\text { Esp knockout (both germline and osteoblast- } \\
\text { specific) }\end{array}$ & Lee et al(9) & $\begin{array}{l}\text { Increased insulin sensitivity Protected from HFD induced } \\
\text { obesity (increased active osteocalcin) }\end{array}$ \\
\hline FoxO1 (osteoblast-specific) Knockout & Rached et al(12) & Lowered blood glucose and increased insulin levels \\
\hline IR (osteoblast-specific) Knockout & $\begin{array}{l}\text { Ferron et al, Feluze et al } \\
(14,15)\end{array}$ & Increased blood glucose and decreased insulin levels \\
\hline GPCR6 & Pi et al(22) & $\begin{array}{l}\text { Increased blood glucose (glucose intolerance), decreased } \\
\text { insulin levels }\end{array}$ \\
\hline TCPTP1 (osteoblast-specific) & Zee et al(11) & $\begin{array}{l}\text { Increased insulin sensitivity (because of increased active } \\
\text { osteocalcin) }\end{array}$ \\
\hline $\begin{array}{l}\text { Osteocalcin knockout (osteoblast- specific) } \\
\text { Osteocalcin knockout (Leydig cell specific, } \\
\text { cyp17i-cre) } \\
\text { Esp (osteoblast- specific) } \\
\text { Esp knockout (Sertoli cells) } \\
\text { GPCR6 (leydig cell specific) }\end{array}$ & Oury et al(23) & $\begin{array}{l}\text { Lowered testis weight, sperm count, testosterone levels } \\
\text { (decreased male fertility) } \\
\text { No effect } \\
\text { Increased testis weight, sperm count, testosterone levels } \\
\text { (Increased male fertility) } \\
\text { No effect } \\
\text { Lowered testis weight, sperm count, testosterone levels } \\
\text { (decreased male fertility) }\end{array}$ \\
\hline
\end{tabular}

